\title{
Genetically Engineered Knock-In and Conditional Knock-In Mouse Models of Cancer
}

\author{
Amy Rappaport ${ }^{1}$ and Leisa Johnson ${ }^{1,2}$ \\ Genentech, Inc., South San Francisco, California 94080
}

\begin{abstract}
Classical transgenic models are useful for quickly gauging the impact of transgene overexpression, but they are limited by the absence of the innate, subtle regulatory elements encoded in introns and other untranslated regions. Moreover, the widespread, high-level expression of oncogenes often leads to tumors that lack the histopathological and acquired genetic features of human cancers. Targeted mutation of endogenous loci, or knock-in (KI) alleles, facilitates more accurate modeling of human tumors by allowing for the expression of mutant alleles under normal physiological regulation. Advanced strategies enable the stochastic activation of such alleles in somatic cells, such that genotypically wild-type cells surround individual mutant cells. More recent technologies, such as site-specific engineered nucleases, have also accelerated the design and implementation of KI strategies. Together, these tools aid in the development of advanced mouse models that better recapitulate the features of human disease.
\end{abstract}

The use of transgenic mouse models of cancer has dramatically increased our knowledge of the genetic interactions and molecular pathways underlying tumor progression. In the 1980s, the advent of recombinant DNA technology and methods for modifying the mouse germline revolutionized the world of cancer biology by enabling the expression of human mutations in the mouse and demonstrating that these genes could drive tumor development (reviewed in Adams and Cory 1991, Introduction: Transgenic Mouse Models_-A Seminal Breakthrough in Oncogene Research [Smith and Muller 2013], and Introduction: Analyses of Tumor-Suppressor Genes in Germline Mouse Models of Cancer [Wang and Abate-Shen 2014]). However, these transgenic mouse models have several limitations. The artificially forced expression of an oncogene or mutant tumor-suppressor gene in a specific cellular compartment, oftentimes at levels exceeding that of the endogenous gene mutation, can promote tumors that do not always reflect the pathobiology of their human disease counterpart. In addition, unlike human cancer mutations, the majority of which arise sporadically, the mutated genes in "classical" transgenic mice are expressed in nearly all cells in which the promoter is active. To generate relevant models of sporadic human cancer, endogenous regulation of the mutant gene must be maintained, including the expression pattern and levels. Moreover, it is critical to faithfully mimic the stochastic and progressive, multistage nature of tumors as well as their innate microenvironment. KI mice, which express an exogenous allele from a specific, targeted locus, were developed to address these fundamental issues. This article will focus on key innovations that have enabled more accurate modeling of human disease, while highlighting specific KI mouse cancer models that exemplify these technological advances.

\footnotetext{
${ }^{2}$ Correspondence: johnson.leisa@gene.com

(C) 2014 Cold Spring Harbor Laboratory Press

Cite this introduction as Cold Spring Harb Protoc; doi:10.1101/pdb.top069799
} 


\section{KI Mouse Technology}

The KI of a mutant allele in the mouse genome can be achieved using homologous recombination (HR) in embryonic stem (ES) cells (Thomas and Capecchi 1987), similar to the methods used to generate conditional knockout mice. However, rather than deleting a portion of a target gene, specific mutations can be introduced into endogenous genes and transmitted throughout the mouse germline. A DNA construct containing the engineered gene of interest (e.g., a mutated oncogene) is flanked by sequences identical to those in the target locus and introduced into ES cells, where homologous sequences align and recombine, thereby introducing the altered gene into an endogenous locus (Fig. 1A). This technology allows for the expression of mutant oncogenes and tumor-suppressor genes from their endogenous promoter, or another promoter of interest, and avoids issues of variability and founder effects that are frequently observed with randomly integrated transgenes.

\section{Modeling Familial Cancer Using Germline KI Mice}

"Classical" transgenic mice, such as those described in Introduction: Transgenic Mouse Models-A Seminal Breakthrough in Oncogene Research (Smith and Muller 2013), have been generated that express gene mutations frequently found in patients with familial/inherited cancer predispositions (reviewed in Adams and Cory 1991). However, the choice of promoter can greatly alter the tumor spectrum and pathology observed in these mice, which often fail to recapitulate key aspects of the human disease. In addition, the cell of origin, and hence the appropriate promoter to drive transgene expression, is not known in many cases. To address this limitation, several KI mouse models have been developed that target the mutation directly to its endogenous gene locus, thereby maintaining innate regulation and more accurately reflecting the expression patterns and levels found in humans carrying the respective germline alteration (Fig. $1 \mathrm{~A}$ and Table 1).

An example of these principles was observed for germline Kit mutations that were identified in familial gastrointestinal stromal tumors (GISTs). Transgenic mice that express these same Kit mutations from a heterologous promoter do not develop GIST, but rather acute lymphoblastic leukemia and lymphomas (Kitayama et al. 1996). In contrast, KI mice that are heterozygous for these familial Kit mutations develop hyperplasia throughout the GI tract, as well as neoplastic lesions with pathology identical to human GISTs (Sommer et al. 2003; Nakai et al. 2007).

In addition, KI mice have also been generated to explore the role of familial mutation of the TP53 tumor-suppressor gene in cancer development. Patients with Li-Fraumeni syndrome, a cancer susceptibility disease in which patients develop a broad range of malignancies, carry a germline mutation in the TP53 tumor-suppressor gene, $80 \%$ of which are missense. This suggests a dominant-negative or gain-offunction role for TP53 missense mutation in cancer. To determine the mechanism(s) of p53 mutant function in vivo, two laboratories have generated KI mouse models of two hot spot missense mutations found in Li-Fraumeni syndrome (Lang et al. 2004; Olive et al. 2004). Mice heterozygous for mutations at either codon 172 or $270\left(p 53^{R 172 H /+}\right.$ or $p 53^{R 270 H /+}$, the equivalent of human $p 53^{R 175 H}$ and $p 53^{R 273 H}$ ) developed more aggressive and metastatic tumor phenotypes than mice heterozygous for $\operatorname{Tr} p 53$ loss $\left(p 53^{+/-}\right)$. In addition, $p 53^{R 172 H /-}$ and $p 53^{R 270 H /-}$ mice acquired a broader histological spectrum of carcinomas compared to $p 53^{-/-}$mice, indicating that mutant $p 53$ has a gain-of-function phenotype in vivo.

These and other KI models recapitulating familial cancers have clearly showed the tumor-promoting effects of various inherited mutations. However, germline mutations account for only $5 \%-10 \%$ of human cancers. To better model the vast majority of somatically arising cancers, more sophisticated techniques have been developed over the years to regulate expression of the disease-associated genetic alteration(s) in a manner that more accurately reflects its spontaneous and limited nature.

\section{ACCURATE MOUSE MODELS OF SOMATIC HUMAN CANCER}

To generate accurate mouse models of somatic human cancer, it is important to maintain endogenous regulation of mutant gene expression in the relevant adult cell/tissue. This requires both temporal and 
Types of Alleles

A KI

Endo $\longdiv { \text { Gene of interest } { } ^ { * } / \mathrm { cDNA } ^ { * } }$

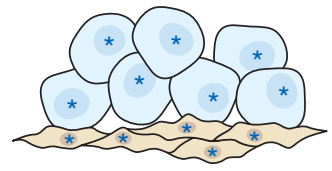

1.

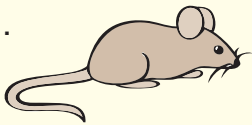

Tissue-specific promoter Recombinase of interest

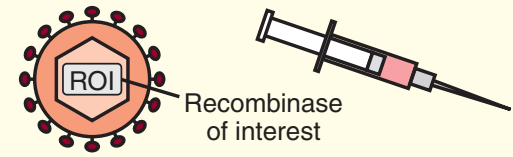

B CKI

1.
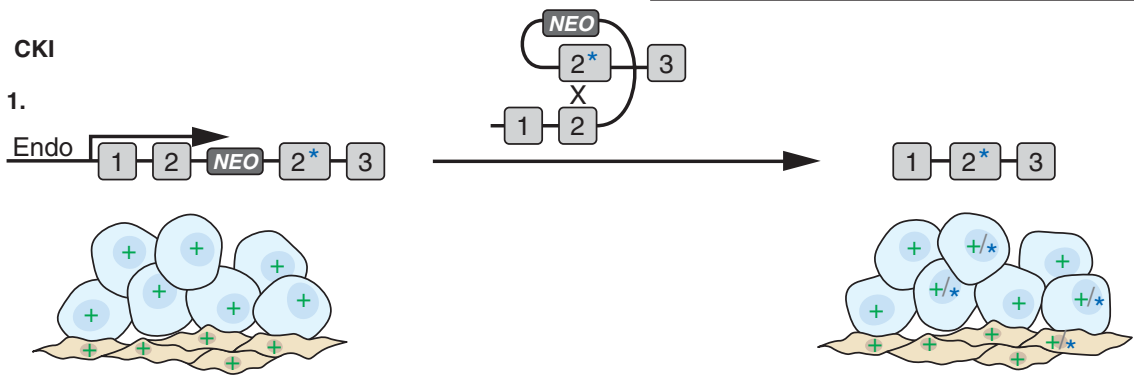

2.

Recombinase of interest

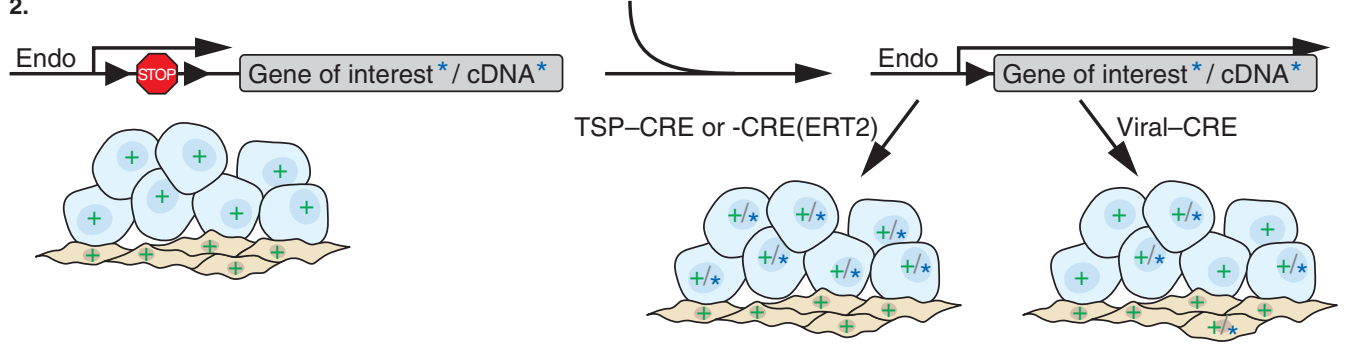

3.
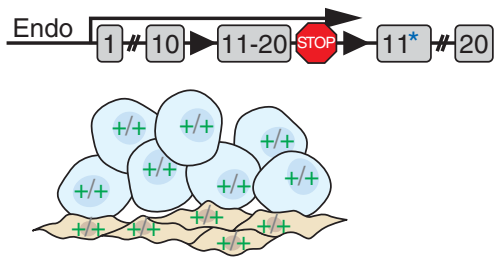

Recombinase of interest

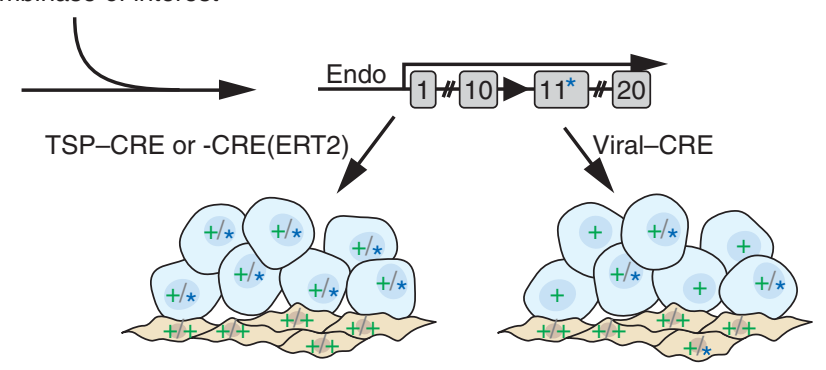

FIGURE 1. Types of knock-in (KI) alleles. (A) Traditional KI alleles target a gene of interest into the endogenous locus (Endo) via homologous recombination. The engineered cDNA (often mutated [ $\left.{ }^{*}\right]$ ) is present in every cell and is expressed from the endogenous promoter in both tumor (blue) and normal (tan) cells. $(B)$ Conditional $\mathrm{KI}(\mathrm{CKI})$ alleles are also targeted to the endogenous locus, but are only expressed following a recombination event. (1) Latent alleles contain a mutated exon but are not expressed due to the presence of a NEO cassette. Following spontaneous recombination in vivo in some cells, the NEO cassette is excised and the mutant gene is expressed in a subset of cells. (2) Insertion of a stop cassette flanked by recombination sites (triangles) before the mutant gene of interest prevents its expression. In mice heterozygous for the conditional KI allele, only a single wild-type allele is expressed (+) before recombination. The stop cassette is excised and the mutant cDNA is expressed following expression of a recombinase of interest (e.g., CRE or CRE(ERT2)). CRE recombinase can either be expressed via a tissue-specific promoter (TSP) or delivered virally (see inset). If a tissue-specific CRE is used, one copy of the mutant cDNA is typically expressed in nearly all of the cells of a given tissue in which the TSP is active (blue cells) and not in the surrounding tissue. If a viral CRE is used, the mutant gene is expressed in a subset of tumor cells and may also (rarely) be expressed in normal tissue. (3) To enable normal levels (two copies, +/+) of gene expression before activation of the mutant allele, a wild-type cDNA fragment flanked by recombination sites and a transcriptional stop cassette is inserted upstream of an intact exon that has been altered to express the mutation of interest. Following delivery of CRE, the normal cDNA fragment and stop cassette are excised and the mutant exon is expressed. (Inset) CKI alleles can be activated in multiple ways. (1) CKI mice are crossed with transgenic mice expressing a recombinase of interest (ROI) from a TSP. (2) Delivery of a virus containing the ROI to a specific tissue of interest (e.g., intranasally or injection into the ducts of the mammary gland). 
TABLE 1. Knock-in mouse models of cancer

\begin{tabular}{|c|c|c|c|c|c|}
\hline $\begin{array}{l}\text { Oncogene/ } \\
\text { TSG }\end{array}$ & Allele & Method of activation & Cooperating allele & Phenotype & Reference(s) \\
\hline KIT & $\begin{array}{l}\text { Kit }{ }^{V 558 \Delta} ; \\
\text { Kit }^{\text {Asp818Tyr }}\end{array}$ & Constitutive & & Gastrointestinal stromal tumors (GISTs) & $\begin{array}{l}\text { Sommer et al. 2003; } \\
\text { Nakai et al. } 2007\end{array}$ \\
\hline TRP53 & $\begin{array}{l}\text { p5 } 3^{\mathrm{R} 172 \mathrm{H}} ; \\
\mathrm{p} 53^{\mathrm{R} 27 \mathrm{OH}}\end{array}$ & Constitutive & & $\begin{array}{l}\text { Wide spectrum of tumors, including sarcomas, lymphomas, and carcinoma in } \\
\text { a variety of tissues, including lung, colon, breast, skin, liver, and pancreas }\end{array}$ & $\begin{array}{l}\text { Lang et al. 2004; } \\
\quad \text { Olive et al. } 2004\end{array}$ \\
\hline \multirow[t]{13}{*}{ KRAS } & $\operatorname{Kras}^{\mathrm{LA}}$ & $\begin{array}{l}\text { Spontaneous } \\
\text { recombination }\end{array}$ & & $\begin{array}{l}\text { Predisposed to lung cancer, thymic lymphoma, and aberrant crypt foci in the } \\
\text { intestine }\end{array}$ & Johnson et al. 2001 \\
\hline & LSL-Kras ${ }^{G 12 V}$ & $\begin{array}{l}\text { CMV-CreERT2+ } \\
\text { systemic 4-OHT }\end{array}$ & & $\begin{array}{l}\text { Pulmonary adenomas; progression to adenocarcinoma at low frequency and } \\
\text { long latency }\end{array}$ & Guerra et al. 2003 \\
\hline & \multirow[t]{9}{*}{ LSL-Kras ${ }^{G 12 D}$} & \multirow[t]{3}{*}{ Intranasal adeno-Cre } & & $\begin{array}{l}\text { Atypical alveolar hyperplasias (AAH) and benign adenomas in the lung, } \\
\text { infrequently progressing to malignant adenocarcinoma }\end{array}$ & $\begin{array}{l}\text { Jackson et al. 2001; } \\
\quad \text { Meuwissen et al. } 2001\end{array}$ \\
\hline & & & p5 $53^{R 270 H}$ & Invasive, metastatic pulmonary adenocarcinoma & Jackson et al. 2001 \\
\hline & & & $L k b 1^{F / F I}$ & $\begin{array}{l}\text { Accelerated tumor progression compared to } \mathrm{Kras}^{\mathrm{G} 12 \mathrm{D}} \text { alone and increased } \\
\text { metastasis, expanded tumor spectrum including squamous cell, } \\
\text { adenosquamous, and large cell carcinoma }\end{array}$ & Ji et al. 2007 \\
\hline & & \multirow[t]{2}{*}{ Pdx1-Cre } & & $\begin{array}{l}\text { Pancreatic intraepithelial neoplasias (PanINs) that progress at low frequency } \\
\text { to invasive and metastatic adenocarcinomas (PDAC) }\end{array}$ & Hingorani et al. 2003 \\
\hline & & & 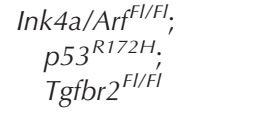 & Increased progression to PDAC & $\begin{array}{l}\text { Aguirre et al. 2003; } \\
\text { Hingorani et al. 2005; } \\
\text { ljichi et al. } 2006\end{array}$ \\
\hline & & K5-Cre*PR1 & & Squamous cell carcinoma of the skin & Caulin et al. 2007 \\
\hline & & Fabpl-Cre; Villin-Cre & $A p c^{2 / 0 \times 14}$ & High-grade colon carcinoma & Haigis et al. 2008 \\
\hline & & Mx1-Cre & & Fatal myeloproliferative disease (MPD) & Braun et al. 2004 \\
\hline & & Intramuscular adeno-Cre & $p 53^{F / / F I}$ & Soft-tissue sarcoma & Kirsch et al. 2007 \\
\hline & \multirow[t]{2}{*}{ FSF-Kras ${ }^{G 12 D}$} & $\begin{array}{l}\text { Intratracheal and } \\
\text { intramuscular adeno- } \\
\text { FlpO }\end{array}$ & $p 53^{F / / F I}$ & Lung tumor and sarcoma development equivalent to $L S L-K r a s{ }^{G 12 D}$ & Young et al. 2011 \\
\hline & & $\begin{array}{l}\text { Intratracheal adeno- } \\
\text { FlpO }\end{array}$ & $N k \times 2-1^{F / / F l}$ & Deletion of Nkx2-1 in established lung tumors altered tumor differentiation & Snyder et al. 2013 \\
\hline \multirow[t]{3}{*}{$B R A F$} & \multirow[t]{3}{*}{$L S L-B r a f^{V 600 E}$} & Intranasal adeno-Cre & $p 53^{F / F I} ; \operatorname{Ink} 4 a / A r f^{F / / F I}$ & $\begin{array}{l}\text { Mixed papillary and solid lung adenomas } \\
\text { Lung adenocarcinomas }\end{array}$ & Dankort et al. 2007 \\
\hline & & Mx1-Cre & & Acute myeloid leukemia (AML) & Mercer et al. 2005 \\
\hline & & $\begin{array}{l}\text { Tyr. CreER } R^{T 2}+\text { topical } 4- \\
\text { OHT to the skin }\end{array}$ & Pten $^{F l / F I}$ & $\begin{array}{l}\text { Benign melanocytic hyperplasias } \\
\text { Invasive metastatic melanoma }\end{array}$ & Dankort et al. 2009 \\
\hline \multirow[t]{2}{*}{ PIK3CA } & \multirow[t]{2}{*}{$\begin{array}{l}\text { Pik3ca } \mathrm{a}^{\mathrm{e} 2 \mathrm{OH} 1047 \mathrm{R}} \\
\text { Pik3ca } \\
\text { LatH1047R }\end{array}$} & MMTV-Cre & & Mammary tumors & $\begin{array}{l}\text { Yuan et al. 2012; } \\
\text { Tikoo et al. } 2012\end{array}$ \\
\hline & & $\begin{array}{l}\text { Intrabursal injection of } \\
\text { adeno-Cre }\end{array}$ & $\operatorname{Pten}^{F / / F I}$ & Ovarian serous adenocarcinomas and granulosa cell tumors & Kinross et al. 2012 \\
\hline$E G F R$ & $\begin{array}{c}\text { CAGGS-LSL- } \\
\text { EGFR }^{\text {vIlI }}\end{array}$ & $\begin{array}{l}\text { Intracranial injection of } \\
\text { adeno-Cre }\end{array}$ & $\begin{array}{c}\text { Ink4a/Arf } f^{F / / F I} \text { and } \\
\operatorname{Pten}^{F / F I}\end{array}$ & Aggressive gliomas & Zhu et al. 2009 \\
\hline
\end{tabular}


spatial control of mutant gene expression in genetically engineered mice. Temporal control of gene expression can be achieved either via stochastic mutation or using inducible KI alleles. In addition, extensive research has focused on enabling induction of the targeted allele in a tissue-specific manner. Here, we will discuss methods to replicate the spontaneous nature of cancer initiating mutations as well as methods to spatially restrict their expression, citing specific examples from the literature (listed in Table 1) to illustrate their application.

\section{Stochastic Mouse KI Models}

One method to generate stochastic mutations in the adult animal takes advantage of "hit and run" gene targeting, which traditionally involves two distinct steps of HR to introduce subtle mutations in ES cells in culture (Hasty et al. 1991). In the first "hit" step, an insertional targeting vector containing the mutant exon is introduced via $\mathrm{HR}$, which leads to a duplication of genomic sequences carrying the mutation and the concomitant integration of a negative-selection marker (Fig. 1B.1). The subsequent "run" step occurs following a rare intrachromosomal recombination event that takes place between the two copies of the targeted exon via either intrachromosomal recombination or unequal sister chromatid exchange. One copy of the exon is then looped out leaving behind either the targeted mutant exon or the original wild-type exon, depending on the location of the crossover event. A study by Johnson et al. (2001) adapted this technique to induce spontaneous expression of an oncogenic Kras ${ }^{G 12 D}$ mutation in vivo. To generate this mouse, a mutant exon was first introduced via HR in vitro (the "hit" step) in the Kras locus in ES cells. Germline transgenic mice were generated carrying this targeted allele and the "run" step allowed to occur stochastically in vivo, so that the Kras ${ }^{G 12 D}$ oncogene was expressed in a fraction of the cells throughout the mouse (Fig. 1B.1). This "latent" mouse model more accurately mimics the early events of tumor development in humans, in which an oncogenic mutation spontaneously occurs in a single cell that is surrounded by wild-type cells. This sporadic cellular context is poorly modeled by classical transgenic strategies. Kras latent mice are strongly predisposed to lung cancer, thymic lymphoma, and aberrant crypt foci in the intestine, nicely demonstrating the powerful oncogenic effects of endogenous $\mathrm{Kras}^{G 12 D}$ in vivo.

\section{Conditional KI Mouse Models}

Although the latent $\mathrm{Kras}^{G 12 D}$ model more closely recapitulates spontaneous oncogene activation, its utility is restricted by the inability to control the frequency, timing, and spatial distribution of the desired recombination event. To address this limitation, significant advances have been made that enable researchers to generate inducible mouse models that provide better control over each of these processes, thereby avoiding issues of embryonic lethality and limiting the occurrence of tumors outside the tissue of interest. First, we will discuss the design of inducible, targeted alleles, followed by a brief overview of various methods for regulation of expression. Then we will describe how conditional systems have been used to accurately model sporadic human cancer, focusing on variations in technology and design.

\section{Removable Transcriptional Termination Stop Elements}

One method that has been very successful and widely adapted is the use of removable transcriptional termination stop elements to control the expression of targeted alleles (Fig. 1B.2). Excision of the STOP cassette, and ensuing expression of the mutant gene, is mediated by inducible and/or lineagespecific recombinases (e.g., Cre or Flp), which allows spatial and temporal control of gene expression (Fig. 1B.2). For clarity, we will describe the construction of these alleles using the Cre/loxP system; however, the same elements apply to other site-specific recombinase systems.

In 1992, Lakso et al. showed that site-specific recombination could activate a dormant transgene in the mouse (Lakso et al. 1992), utilizing the Cre recombinase derived from bacteriophage P1 and its cognate, site-specific loxP recognition sites. Lakso et al. generated a transgene in which they placed a 
synthetic transcriptional termination STOP sequence, consisting of the carboxy-terminal sequence of the yeast His3 gene and the SV40 virus polyadenylation signal, between the $\alpha$-crystallin lens-specific promoter and the SV40 virus large tumor antigen (TAg), thereby effectively suppressing TAg expression. To render this allele inducible, the STOP sequence was flanked on either side by loxP sites. The loxP sites consist of 13-bp inverted repeats separated by an 8-bp spacer. When two loxP sites are placed in the same orientation, Cre recombinase will bind and excise the intervening sequences. Transgenic mice carrying this allele in combination with a cytomegalovirus (CMV) promoter-driven Cre recombinase showed spatially restricted TAg expression and the development of lens tumors (Lakso et al. 1992).

Subsequent studies improved upon this technology by targeting the lox-STOP-lox (LSL) cassette directly to the endogenous locus (Fig. 2A), and by introducing additional genetic elements to eliminate "leakiness" by further damping expression in the unrecombined state. One of the most widely used transcriptional STOP cassettes was developed by Jackson et al. (2001) to mediate conditional expression of a $\mathrm{Kras}^{G 12 D} \mathrm{KI}$ allele. In this example, the removable STOP element was placed between the endogenous Kras promoter and a mutant Kras exon 1 (Fig. 2A). This STOP cassette contained a drug selectable marker driven by the constitutive Pgk promoter and transcribed in the opposite direction as Kras, thereby enabling the selection of correctly targeted ES cells. Several additional design elements were included in this STOP element to prevent leaky expression of the mutant gene before its removal. First, the drug selection cassette was followed by multiple SV40-derived poly(A) sequences to terminate transcription of the targeted allele from its endogenous promoter.

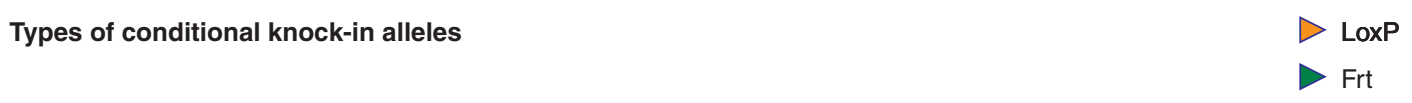

A

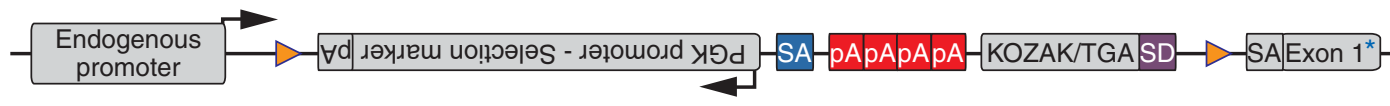

B

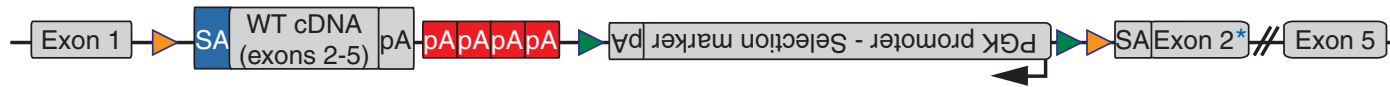

C

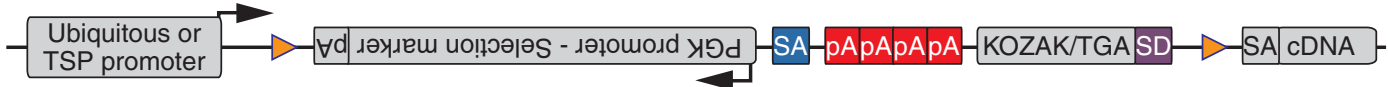

FIGURE 2. Design of conditional KI alleles. (A) The lox-stop-lox (LSL) cassette is targeted to an endogenous locus and prevents expression of the mutant gene. A Pgk promoter-driven selectable marker is transcribed in the opposite direction and has its own poly(A) signal ( $\mathrm{pA}$ - gray). This is followed by a strong splice acceptor sequence (SA-blue), which serves as a $5^{\prime}$ splice donor trap to prevent unwanted splicing to the mutant exon 1 splice acceptor (SA—gray) before the excision of the STOP cassette. Multiple SV40-derived poly(A) sequences (pA-red) stop transcription. A Kozak-stop codon sequence blocks translation and a splice donor site (SD) immediately downstream of this "miniexon" helps ensure that any splicing to the exon $1 \mathrm{SA}$ would be translationally terminated. Following recombination at the loxP sites (orange triangles), the LSL cassette is excised and the mutant gene is expressed from the endogenous promoter. An asterisk denotes targeted mutation of interest. $(B)$ The LSL cassette is targeted within an endogenous gene. Here, the LSL cassette contains a cDNA fragment encoding a portion of the wild-type gene, including the gene's endogenous poly(A) signal, which is immediately followed by multiple additional poly(A) sites to prevent transcriptional readthrough of the mutant exon. To favor splicing to this CDNA fragment versus the downstream endogenous and mutated exon, a strong SA site is also included. A Pgk promoter-driven selectable marker is included to facilitate the identification of correctly targeted ES cells and should be excised in vitro following recombination at the Frt sites (green triangles). This design maintains expression of the wild-type gene from the endogenous promoter before Cre-mediated excision of the STOP cassette. An asterisk denotes targeted mutation of interest. (C) An LSL cassette followed by the cDNA of the gene of interest or a reporter allele can be targeted to a ubiquitous or TSP. 
Second, a strong splice acceptor was placed $5^{\prime}$ of the poly(A) signals to serve as a $5^{\prime}$ splice donor trap, thereby preventing undesired splicing to the start of exon 1 and circumvention of the STOP element. Third, a Kozak sequence followed by a stop codon was added to abort translation of any transcripts before the mutant exon; however, this is an added safety feature that has not been validated. Finally, an additional splice donor site was incorporated immediately downstream of the stop codon to ensure that any aberrant splicing to exon 1 would be translationally terminated. Following recombination and excision of the STOP cassette, these elements are removed and transcription from the endogenous promoter proceeds normally through the mutated exon 1 and remaining wild-type exons (Fig. 1B.2). Although variations in the design of the STOP element exist, it is important to consider features such as those described above to minimize undesirable transcription and/or translation of the mutant gene before excision.

\section{Maintenance of Wild-Type Gene Expression}

A significant limitation of the conditional KI allele described above is that there is no expression of the targeted allele in the absence of Cre recombination. This can result in haploinsufficient levels of the wild-type protein in heterozygous mice or no expression in mice homozygous for the engineered allele, both of which can be deleterious during development or have unintended side effects. In human cancer cells, one copy of a wild-type gene is first converted to a mutant version in the adult tissue. To more faithfully recapitulate this, normal levels of the wild-type gene should be preserved before the desired recombination event, at which time the mutant gene should be expressed at physiological levels and subject to normal patterns of splicing. To achieve this, several laboratories have designed targeted alleles that incorporate a mini-cDNA containing the wild-type exons within the LSL cassette, followed by the mutant exon (Figs. 1B.3 and 2B). In this case, the STOP element is the wild-type cDNA itself, followed by multiple poly(A) signals. The LSL cassette still contains a Pgk promoter-driven selectable marker to enable the detection of correctly targeted ES cells; however, this feature is deleted in vitro using an alternative recombinase (e.g., Flp) before generating mice. Following Cre recombinase expression in the adult mouse, the wild-type cDNA is excised and the mutant gene product expressed (Fig. 1B.3). These types of alleles have been created for a variety of different oncogenic mutations for which maintenance of endogenous gene expression and signaling is critical, including $\mathrm{Braf}^{\mathrm{V6OOE}}$ and $\mathrm{Pik}_{\mathrm{ca}} \mathrm{H1O47R}^{\mathrm{H}}$ (Table 1) (Mercer et al. 2005; Dankort et al. 2007; Yuan et al. 2012).

\section{METHODS FOR REGULATING TEMPORAL AND SPATIAL RECOMBINATION/ACTIVATION}

Inducible Recombinase Expression

The strength of conditional KI mice is the ability to both temporally and spatially control the activation of the mutant gene. This can be achieved by a variety of regulatory methods (Fig. 1, inset).

One method to temporally regulate expression of a mutant allele is to use an inducible recombinase. Several laboratories have generated transgenic mice expressing the Cre recombinase fused to a mutated ligand-binding domain of the human estrogen receptor $\left(\mathrm{ER}^{\mathrm{T} 2}\right)$, which binds to the synthetic steroid tamoxifen, but not estrogen (Brocard et al. 1997). The $\mathrm{ER}^{\mathrm{T} 2}$ moiety restricts Cre to the cytoplasm; treatment with 4-hydroxy-tamoxifen (4-OHT) enables Cre to move into the nucleus, excise the STOP cassette, and activate gene expression (Fig. 1B.2 and 1B.3). A variety of CreER ${ }^{\mathrm{T} 2}$-expressing transgenic and $\mathrm{KI}$ mice have been generated which drive the fusion protein from different ubiquitous promoters. For example, to achieve sporadic expression in a wide range of adult (or embryonic) tissues and cell types, Guerra et al. generated a KI mouse strain that targeted the $\mathrm{CreER}^{\mathrm{T} 2}$ fusion to the RNA polymerase II large subunit locus. This mouse was subsequently bred to a strain containing a conditional, LSL-regulated $\mathrm{Kras}^{G 12 \mathrm{~V}}$ allele (Guerra et al. 2003). Systemic treatment of postnatal (P10) Kras ${ }^{G 12 \mathrm{~V} / \mathrm{t}}$; RERTn ${ }^{E R T 2 /+}$ mice with 4 -OHT led to expression of mutant $\mathrm{Kras}^{\mathrm{Gl} 2 \mathrm{~V}}$ in $5 \%-15 \%$ of cells in most tissues. 


\section{Tissue-Specific Expression}

To restrict gene expression to a specific cell type, expression of the recombinase can be placed under the control of a TSP. A wide variety of tissue-specific Cre- and CreER ${ }^{\mathrm{T} 2}$-expressing mouse strains have been generated. For example, by crossing the $L S L-K r a s{ }^{G 12 D}$ mice described above to transgenic mice containing Cre under the control of a pancreatic-restricted promoter (e.g., Pdxl-Cre), Kras ${ }^{G 12 D}$ expression can be preferentially restricted to pancreatic progenitor cells. These mice develop ductal lesions that recapitulate the full spectrum of human pancreatic intraepithelial neoplasias (PanINs), which progress at a low frequency to invasive and metastatic adenocarcinomas (PDAC) (Hingorani et al. 2003). Similarly, mouse models of squamous cell carcinoma of the skin, colon cancer, and hematopoietic malignancies have been generated by crossing conditional KI mice containing the LSL$\mathrm{Kras}^{G 12 D}$ allele with the appropriate tissue-specific, Cre-expressing strain (Braun et al. 2004; Caulin et al. 2007; Haigis et al. 2008; Hung et al. 2010).

In each of the above scenarios (ubiquitous vs. TSP), one also has the ability to regulate, to some degree, the number of recombination events, either through the applied dosage of 4-OHT and/or the choice of tissue-specific CreER ${ }^{\mathrm{T} 2}$ transgenic strain. In the latter case, insertion site effects can further influence the percentage of cells in which $\mathrm{CreER}^{\mathrm{T} 2}$ is expressed within a given tissue.

Viral Delivery of Recombinases

Another method to induce gene activation in both a temporally and spatially regulated manner is via viral delivery of the recombinase(s) using adenovirus or lentivirus. The method and site of delivery (e.g., inhalation or injection to a particular organ) allows control over the tissue where the gene is induced and, in addition, serves to restrict gene expression to a small subset of cells, depending on both the applied viral dose and the virus' tropism (Fig. 1B.2 and 1B.3). For example, the Jacks and Berns laboratories both independently developed mouse models in which oncogenic Kras activation was targeted to the lung. In both cases, the conditional alleles of oncogenic Kras ( $\mathrm{Kras}^{G 12 D}$ ) were similarly designed to contain an LSL cassette (Jackson et al. 2001; Meuwissen et al. 2001), and expression of mutant $\mathrm{Kras}^{G 12 D}$ selectively occurred following intra-nasal delivery of a recombinant adenovirus expressing the Cre-recombinase (Adeno-Cre) and excision of the STOP cassette. Atypical alveolar hyperplasias (AAH) could be detected in the lungs by $\sim 4$ wk postinfection; over time, these lesions were capable of progressing into benign adenomas and, more infrequently, malignant adenocarcinomas.

Sequential and/or Spatial Regulation of Multiple Alleles

The various methods described above for regulating gene expression can be combined to temporally and/or spatially separate the in/activation of more than one mutant allele. By utilizing both the Cre/lox and $\mathrm{Flp} / \mathrm{frt}$ recombinase systems, multiple mutant alleles can be combined and conditionally regulated via unique recombinases, whose expression may be differentially controlled over time and/or in distinct cellular compartments (Perez-Mancera et al. 2012). For example, the Jacks laboratory recently generated an alternative conditional $\mathrm{Kras}^{\mathrm{G} 12 \mathrm{D}} \mathrm{KI}$ allele that is preceded by a frt-STOP-frt (FSF) cassette (Young et al. 2011), which can be activated via a tissue-specific Flp strain or through viral delivery of an Flp recombinase. Activation or deletion of a second gene of interest (e.g., a candidate drug target or regulator of differentiation or metastasis) can subsequently be induced via regulated Cre expression (Snyder et al. 2013). The use of a ubiquitous CreER ${ }^{\mathrm{T} 2}$-expressing strain (e.g., Rosa26-CreER ${ }^{T 2}$ or $C A G G s-C r e E R^{T 2}$ ) will induce mutant allele expression globally, thereby mimicking the effect of a systemically delivered targeted inhibitor. Alternatively, the secondary alteration may be induced in a tissue/cell-restricted manner by utilizing a tissue-specific Cre or CreER ${ }^{T 2}$ mouse strain, especially if one is interested in interrogating distinct tumor cell compartments (e.g., stromal or immune cells). This system can be even more finely controlled by utilizing an FSF-regulated, ubiquitous $C r e E R^{T 2}$ strain. In this scenario, Cre, and thus the second gene of interest, are expressed only in cells that have already been activated by Flp (i.e., tumor cells expressing $\mathrm{Kras}^{G 12 D}$ ) and following exposure to 4-OHT, thereby mimicking the stepwise progression of mutations in tumor development. 
Importantly, these models and approaches more closely mimic both the spontaneous nature and multistep pathogenesis observed in human cancer and have extensively been used to provide mechanistic insight into the genetic and molecular pathways underlying cancer progression as well as an autochthonous setting for assessing preclinical efficacy and mechanisms of therapeutic response and resistance.

\section{Overexpression of an Oncogene}

Although maintenance of endogenous gene regulation is typically desired, sometimes the overexpression of a gene of interest may be advantageous to accurately model an oncogene that is often overexpressed and/or amplified in human cancer. For example, two different mouse models carrying conditional mutant Egfr alleles knocked into the endogenous locus have been generated, and in both cases, neither allele led to the development of lung cancer following EGFR activation, even when the alleles were rendered homozygous or combined with numerous conditional tumor suppressor alleles (L Johnson, unpubl.; T Jacks, pers. comm.). These data suggest that amplification/overexpression of Egfr mutants is required for eliciting their oncogenic effects in mice.

One potential method to attain overexpression of a gene of interest is to target a conditional allele to a ubiquitously expressed locus like Rosa26 or CollA, either with or without an enhanced promoter element (e.g., CAGGS). Ideally, this locus should be inert, such that its disruption has no deleterious effects on the animal and does not cooperate with your gene of interest in tumorigenesis. Here, the design of the transcriptional STOP element is similar (Fig. 2C), and should include many of the feature discussed above. Zhu et al. (2009) successfully used this approach to generate a KI model of glioblastoma (GBM) that was driven by mutant EGFR overexpression. They inserted an LSL-EGFRvIII allele driven by the ubiquitous CAGGS promoter into the CollA locus. Activation of this mutant EGFR allele specifically in the mouse striatum was mediated by intracranial injection of Adeno-Cre. Although overexpression of EGFRvIII alone was insufficient to induce tumorigenesis in this tissue, concomitant loss of Ink4a/Arf and Pten cooperated to give highly aggressive gliomas with $100 \%$ penetrance.

\section{Reversible Gene Regulation}

Although the Cre/loxP recombination system facilitates both temporal and spatial control of the expression of the desired gene product, these effects are permanent-that is, once the LSL cassette has been excised, expression can no longer be regulated. Several mechanisms for mediating reversible gene expression have also been developed and used in KI mouse generation, including alternative sitespecific recombinase systems (e.g., Flp/Frt) whose reversibility is dictated by the orientation of their cognate recognition sequences.

In addition, estrogen receptor ligand-binding domains, similar to that in the $\mathrm{CreER}^{\mathrm{T} 2}$ fusion protein described above, can also be directly conjugated to oncogenes. A KI mouse expressing an MYC-ER fusion protein targeted to the Rosa26 locus has been generated. Importantly, this locus is expressed at levels comparable to that of endogenous MYC and enables reversible expression of physiological levels of MYC following tamoxifen treatment (Murphy et al. 2008).

Similarly, tetracycline-inducible transgenes have extensively been used to generate inducible mouse models. In this case, a targeting construct containing the gene of interest under the control of the tet-response element (TRE) is knocked-in to either its endogenous or an inert locus. Activation of the TRE requires coexpression of a tet transactivator along with treatment with tetracycline or a tetracycline analog. Tissue-specific expression can be achieved by placing control of the tet transactivator under a TSP. Although both of these methods enable reversible gene expression, they are limited in that they do not maintain endogenous gene regulation.

\section{FACILITATION OF DETECTION}

Given the vast number of ways one could combine the above technologies to enable precise control over the timing and location of gene induction, methods to facilitate the detection of gene expression 
are extremely useful. For example, reporter genes (e.g., $\beta$-geo or fluorescent markers) are often used to report expression of the mutant gene product. There are two methods for reporter-gene expression. First, one can link the expression of the reporter directly to that of the targeted allele using sequences that enable bicistronic gene expression, such as an internal ribosomal entry sequence (IRES) or viral 2A-peptide, to enable expression of the reporter on the same transcript as the mutant gene (Martin et al. 2006; Kim et al. 2011). In this way, one can determine the location of gene expression by immunohistochemistry or flow cytometry. However, it is important to note that these types of features may alter the level of expression of the upstream gene, which could inadvertently affect observed phenotypes.

Alternatively, several reporter mouse strains have been generated by targeting a conditional reporter gene to a ubiquitous and inert locus (e.g., Rosa26). Here, a STOP cassette, similar to those described previously, is placed between the promoter and reporter gene (Fig. 2C). These mouse strains are crossed with mice that are also containing a similar recombinase-regulated conditional allele, such that following recombinase expression, the STOP cassettes of both alleles are excised and cells expressing the mutant allele are concomitantly labeled with the reporter. Alternatively, to avoid disruption of an endogenous gene, the reporter gene can be targeted to a ubiquitous locus using an IRES or $2 \mathrm{~A}$ peptide, thereby enabling expression of both the endogenous and reporter genes. The use of reporter mice is discussed in further detail in Introduction: Imaging Mouse Cancer Models In Vivo Using Reporter Transgenes (Lyons et al. 2013).

\section{THE FUTURE OF KI MOUSE MODELS}

$\stackrel{\infty}{\circ} \stackrel{0}{\circ}$ Improved Technologies for Generation of Mice

The KI mouse models described above are powerful tools for elucidating the mechanisms of tumor initiation, progression, metastasis, therapeutic response, and resistance. However, these models are not without their caveats. For example, the generation of KI mice with multiple allelic combinations requires lengthy breeding strategies that are often time-, cost-, and labor-intensive. It is also challenging to produce large, synchronized cohorts of mice, particularly when dealing with multiallelic colonies, as only a subset of progeny mice are of the desired genotypes. These issues ultimately limit the broader use and application of genetically engineered mouse models (GEMMs) for high-throughput screening and drug testing. However, recent technological advancements have been developed that increase the speed at which new models and large cohorts can be generated.

\section{Site-Specific Engineered Nuclease Technology for Genetic Engineering of Animals}

The development of site-specific engineered nucleases has enabled more rapid and efficient generation of animal models with gene-targeted deletions or mutations. These hybrid molecules are composed of a DNA-binding domain (DBD) targeted to a specific sequence in the genome and a nuclease cleavage domain that induces site-specific double-strand breaks (DSBs) (Fig. 3). Historically, this has been achieved utilizing zinc-finger nucleases (ZFNs), which consist of a zinc-finger-derived DBD linked to the nuclease domain of the FokI restriction enzyme. However, more recently, the use of transcription activator-like effector nucleases (TALENs), which are similar but use a DBD derived from TAL effector proteins (reviewed in Carroll 2011; Joung and Sander 2012; Mussolino and Cathomen 2012), has gained traction. Expression of these proteins in cell lines or embryos, either via transfection of plasmid DNA or via microinjection of mRNA, generates site-specific DSBs and DNA repair by nonhomologous end joining (NHEJ), which, in turn, generates short deletions or insertions of varying length at the cleavage site. This technique has been used extensively to generate inactivating mutations in specific genes in human cell lines, including pluripotent stem cells (Lombardo et al. 2007; Hockemeyer et al. 2011), as well as to generate transgenic knockout animals in a variety of species, including mice (Carbery et al. 2010; reviewed in Rémy et al. 2009). In addition, several laboratories have showed that the addition of a donor DNA template induces DNA repair by HR and insertion of foreign DNA 
Downloaded from http://cshprotocols.cshlp.org/ on April 26, 2023 - Published by

A

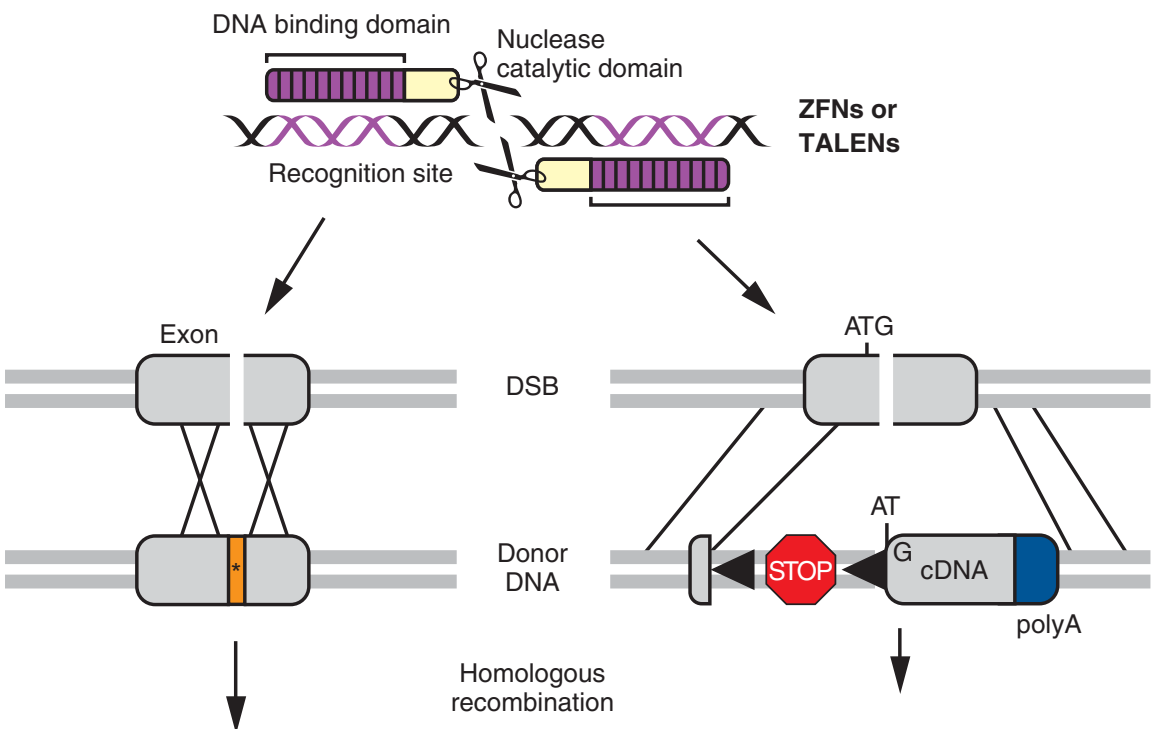

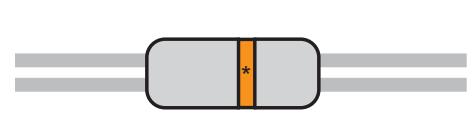

KI

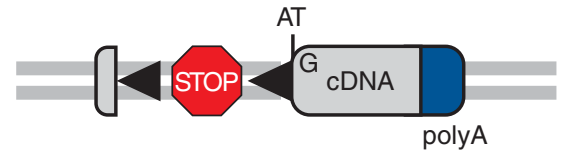

CKI

B

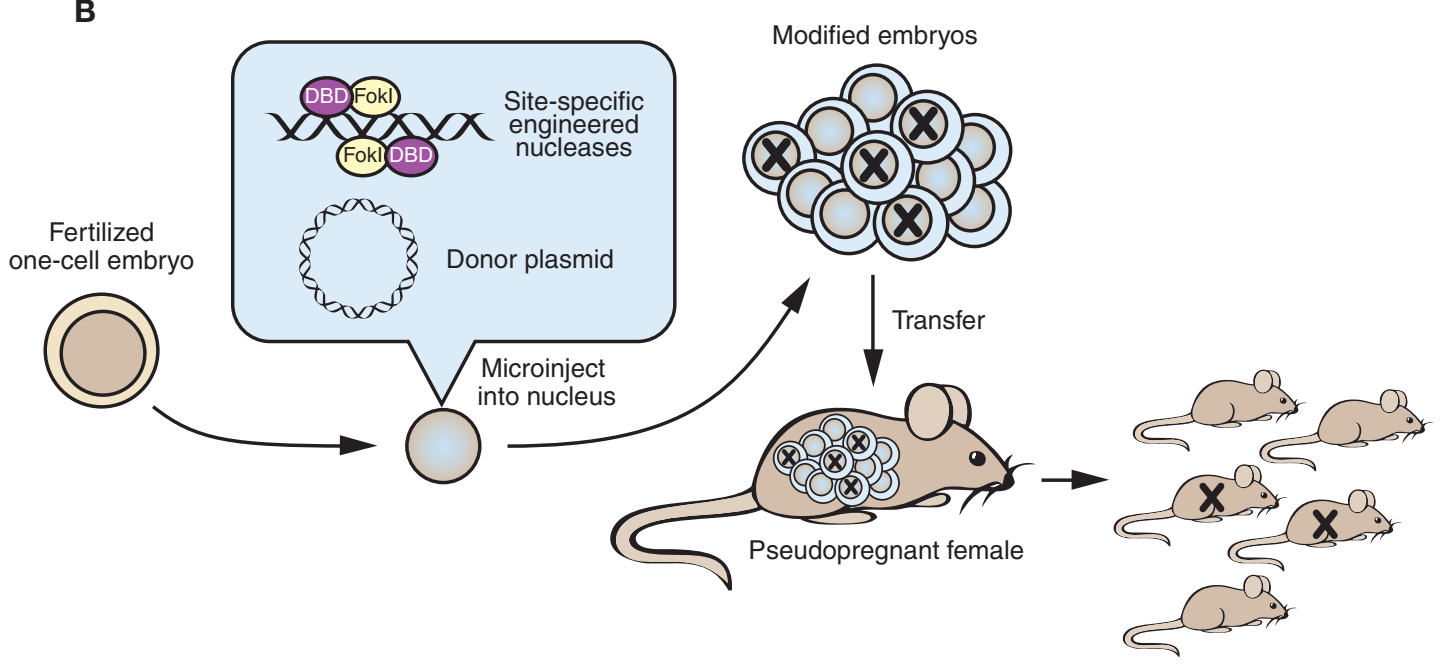

FIGURE 3. Site-specific engineered nucleases for the rapid generation of KI mice. (A) Zinc-finger nucleases (ZFNs) or transcription activator-like effector nucleases (TALENs) contain a double-stranded break (DSB) that complements a recognition site in the genome. Following dimerization, the nuclease catalytic domain induces a DBD at a specific site in the gene of interest. (Left panel) To generate a KI allele, the donor DNA contains a mutation, which is flanked by sequence homologous to the target gene. Following HR, the mutated sequence is integrated into the genome. (Right panel) To generate a conditional KI allele (CKI), the donor DNA contains a transcriptional stop element flanked by recombination sites (triangles), followed by a mutant cDNA. This cassette is then flanked by homologous sequence. Following recombination, the conditional allele is integrated, replacing the endogenous exon. Recombinase-mediated excision generates the ATG needed to ensure expression of the mutated cDNA. (B) To generate KI mice, a sitespecific engineered nuclease containing a DBD fused to a nuclease catalytic domain (e.g., from the Fokl restriction enzyme) is injected into the nucleus of a fertilized embryo along with a donor plasmid containing the mutant sequence flanked by homologous sequence matching the target locus. Modified embryos are then transferred into pseudopregnant female mice, which give birth to mice containing the modified allele in their germlines. 
sequences into a targeted locus (Fig. 3A). Using this approach, researchers have successfully targeted site-specific modifications in human cells as well as rat and mouse embryos, thereby enabling the generation of KI mice (Moehle et al. 2007; Meyer et al. 2010; Cui et al. 2011).

\section{Design and Selection of Site-Specific Nucleases}

The Fok1 nuclease requires dimerization for activation, thus targeting a locus requires the design of a pair of sequence-specific molecules. The DNA binding of ZFNs is determined by highly variable zincfinger domains, the specificity of which are dependent on neighboring domains. This makes their design challenging, requiring complex algorithms and selection. In addition, functional ZFNs may not be available for every loci of interest. In contrast, TALENs use Tal-effector binding domains, which are highly modular and specific to a given base pair. This makes them more tractable as they can easily be designed to target virtually any sequence in the genome (Joung and Sander 2012; Mussolino and Cathomen 2012). TALEN pairs appear to have a higher success rate than ZFNs but have yet to be as extensively characterized in as many species or in vivo applications (Cade et al. 2012; Reyon et al. $2012 \mathrm{~b}$ ). Although mutation efficiency varies by cell type, current studies suggest that both ZFNs and TALENs commonly induce mutation in $>10 \%$ of cells, and a rate of $50 \%$ is typically observed in mouse embryos. To date, both ZFNs and TALENs have extensively been tested on a number of endogenous gene loci. In addition, ZFNs have been successfully used to target many types of sequences, including noncoding RNA (Gutschner et al. 2011), intronic regions (Cristea et al. 2011), and promoter and enhancer regions (Isalan et al. 2001); TALENs are currently being tested in a similar manner.

ZFNs are commercially available, but can also be designed using open-source platforms (www .zincfingers.org) and synthesized using standard cloning techniques (Maeder et al. 2008; Sander et al. 2011a). TALENs are also commercially available, but are much more straightforward to design and synthesize because of their modular nature. Methods using both standard and high-throughput cloning techniques have recently been developed and are publicly available (www.talengineering. org) (Huang et al. 2011; Sander et al. 2011b; Weber et al. 2011; Reyon et al. 2012a,b).

One potential issue with site-specific nucleases is off-target mutation. The requirement for Fok1 dimerization and two binding sites greatly increases specificity; however, small genes and repetitive sequences remain difficult to target successfully. High levels of off-target mutation can also lead to cytotoxicity. Several laboratories have developed strategies to evaluate the specificity of ZFNs as well as reduce cytotoxicity (Gabriel et al. 2011; Pattanayak et al. 2011). The specificity of TALENs can be uniquely enhanced by increasing the length of the TALEN-binding domain (Reyon et al. 2012b). However, as off-target mutations may still occur, it is important to evaluate more than one mutant clone for a given target.

\section{Generation of Mice}

The expression of these molecules via microinjection of mRNA in mouse zygotes (or cells) leads to site-specific alterations in $\sim 50 \%$ of embryos. Zygotes are then transferred into pseudopregnant females, generating F1 mice that in theory have germline mutation at the target site in one allele (Fig. 3B). In practice, however, this depends on how long the ZFN or TALEN is active, as they can induce the DSB at later divisions, which can lead to chimeric mice having multiple different mutant alleles. This is an important consideration, because mice must be screened for mutations and then bred for the desired mutant allele to obtain a pure strain. Alternatively, this can enable the production of compound knockout mice in the first generation, thereby allowing for rapid screening for phenotypic effects. This approach has been used successfully to generate both heterozygous and homozygous knockout mice (Carbery et al. 2010). Importantly, this technique can be applied to any mouse strain, which can circumvent the lengthy backcrossing typically required for transgenic mice, as well as in other species (e.g., rats, rabbits).

In addition to the generation of small inactivating deletions and insertions by NHEJ, novel DNA sequences can be integrated in a target site via HR (Fig. 3A, left panel). This could be used to generate a 
specific point mutation or introduce a reporter gene. This technique has successfully been used to insert a 12-bp tag, a 900-bp open reading frame (ORF), and a 1.5-kb promoter transcription unit into human cells (Moehle et al. 2007). Simultaneous studies in the Kuhn and Weinstein laboratories have showed that transgenic KI mice can be generated using ZFNs to integrate functional reporter cassettes, although mutation efficiency is much lower than for NHEJ indels (Meyer et al. 2010; Cui et al. 2011). To further expand this technology, efforts are currently underway to insert larger sequences of DNA that will enable the generation of conditional KI and knockout alleles via the insertion of site-specific recombination sites (e.g., loxP). For example, one could insert a mutant exon flanked by two loxP sites into the endogenous locus and generate a conditional KI (Fig. 3A, right panel). One technical concern with larger sequences is that crossover can occur at multiple places within the fragment being inserted, thereby generating alleles with only partial integration. One strategy to overcome these unwanted crossovers is to introduce silent mutations throughout the donor sequence, thereby decreasing overall homology with the endogenous sequence and, hence, rates of recombination (S Warming, pers. comm.). Another group has also recently altered the nucleases to favor rates of HR versus NHEJ (Ramirez et al. 2012).

\section{Future Directions for Engineering Mice Using Genome Editors}

The speed at which KI mice can be generated using site-specific engineered nucleases promises to broaden the application and scope of GEMMs for disease research in the near future. One current limitation is the lack of inducible forms of ZFNs and TALENs, which confines modifications to nonessential genes. It would be highly advantageous to enable inducible expression of these nucleases in the adult mouse via integration of the specific nuclease paired sequences into an inert locus under the control of systems like Cre/loxP or tet. In this context, expression of the ZFN or TALEN pairs could be induced in the adult mouse, generating inactivation of a candidate tumor-suppressor or target gene in a subset of cells within a tissue or tumor.

Recent work has also explored the fusion of site-specific DNA-binding proteins to other types of effector molecules, such as transcriptional activators, repressors, and chromatin remodelers. There are many possible applications for the use of these fusion proteins to govern gene expression in inducible mouse models, including modeling overexpression of amplified oncogenes. Moreover, these domains have been engineered to enable regulation of gene expression by small molecules (reviewed in Davis and Stokoe 2010). This technology was showed by generating inducible expression of human VEGF from the endogenous locus in cell lines (Pollock et al. 2002). In the future, this technology could be readily adapted to enable the rapid generation of KI mice with inducible gene expression.

The development of DNA recombinant technology and new methods for conditional control of gene expression has enabled the generation of new mouse models that more accurately recapitulate human disease. These mice have been used successfully to elucidate mechanisms of tumor initiation, progression, metastasis, therapeutic response, and resistance. With further technological advancements, GEMMs will be more widely integrated into the pharmaceutical development pipeline, and be used as a preclinical platform to help guide and improve human clinical trial design and outcome. Large-scale screening efforts currently underway in GEMMs will also produce novel drug targets and further elucidate mechanisms influencing tumor progression and therapeutic response.

\section{ACKNOWLEDGMENTS}

We thank Allison Bruce for the excellent graphics she generated to accompany this article. 


\section{REFERENCES}

Adams JM, Cory S. 1991. Transgenic models of tumor development. Science 254: 1161-1167.

Aguirre AJ, Bardeesy N, Sinha M, Lopez L, Tuveson DA, Horner J, Redston MS, DePinho RA. 2003. Activated Kras and Ink4a/Arf deficiency cooperate to produce metastatic pancreatic ductal adenocarcinoma. Genes Dev 17: 3112-3126.

Braun BS, Tuveson DA, Kong N, Le DT, Kogan SC, Rozmus J, Le Beau MM, Jacks TE, Shannon KM. 2004. Somatic activation of oncogenic Kras in hematopoietic cells initiates a rapidly fatal myeloproliferative disorder. Proc Natl Acad Sci 101: 597-602.

Brocard J, Warot X, Wendling O, Messaddeq N, Vonesch JL, Chambon P, Metzger D. 1997. Spatio-temporally controlled site-specific somatic mutagenesis in the mouse. Proc Natl Acad Sci 94: 14559-14563.

Cade L, Reyon D, Hwang WY, Tsai SQ, Patel S, Khayter C, Joung JK, Sander JD, Peterson RT, Yeh JR. 2012. Highly efficient generation of heritable zebrafish gene mutations using homo- and heterodimeric TALENs. Nucleic Acids Res 40: 8001-8010.

Carbery ID, Ji D, Harrington A, Brown V, Weinstein EJ, Liaw L, Cui X. 2010. Targeted genome modification in mice using zinc-finger nucleases. Genetics 186: 451-459.

Carroll D. 2011. Genome engineering with zinc-finger nucleases. Genetics 188: 773-782.

Caulin C, Nguyen T, Lang GA, Goepfert TM, Brinkley BR, Cai W-W, Lozano G, Roop DR. 2007. An inducible mouse model for skin cancer reveals distinct roles for gain- and loss-of-function p53 mutations. J Clin Invest 117: 1893-1901.

Cristea S, Gregory PD, Urnov FD, Cost GJ. 2011. Dissection of splicing regulation at an endogenous locus by zinc-finger nuclease-mediated gene editing. PLoS One 6: e16961.

$\div$ Cui X, Ji D, Fisher DA, Wu Y, Briner DM, Weinstein EJ. 2011. Targeted integration in rat and mouse embryos with zinc-finger nucleases. Nat Biotechnol 29: 64-67.

Dankort D, Filenova E, Collado M, Serrano M, Jones K, Mcmahon M. 2007. A new mouse model to explore the initiation, progression, and therapy of BRAFV600E-induced lung tumors. Genes Dev 21: 379-384.

Dankort D, Curley DP, Cartlidge RA, Nelson B, Karnezis AN, Damsky WE Jr, You MJ, DePinho RA, McMahon M, Bosenberg M. 2009. Braf $^{\mathrm{V} 600 \mathrm{E}}$ cooperates with Pten loss to induce metastatic melanoma. Nat Genetics 41: 544-552.

Davis D, Stokoe D. 2010. Zinc finger nucleases as tools to understand and treat human diseases. BMC Med 8: 42 .

Gabriel R, Lombardo A, Arens A, Miller JC, Genovese P, Kaeppel C, Nowrouzi A, Bartholomae CC, Wang J, Friedman G, et al. 2011. An unbiased genome-wide analysis of zinc-finger nuclease specificity. Nat Biotechnol 29: 816-823.

Guerra C, Mijimolle N, Dhawahir A, Dubus P, Barradas M, Serrano M, Campuzano V, Barbacid M. 2003. Tumor induction by an endogenous K-ras oncogene is highly dependent on cellular context. Cancer Cell 4: $111-120$.

Gutschner T, Baas M, Diederichs S. 2011. Noncoding RNA gene silencing through genomic integration of RNA destabilizing elements using zinc finger nucleases. Genome Res 21: 1944-1954.

Haigis KM, Kendall KR, Wang Y, Cheung A, Haigis MC, Glickman JN, Niwa-Kawakita M, Sweet-Cordero A, Sebolt-Leopold J, Shannon KM, et al. 2008. Differential effects of oncogenic K-Ras and N-Ras on proliferation, differentiation and tumor progression in the colon. Nat Genet 40: 600-608.

Hasty P, Ramirez-Solis R, Krumlauf R, Bradley A. 1991. Introduction of a subtle mutation into the Hox-2.6 locus in embryonic stem cells. Nature 350: 243-246.

Hingorani SR, Petricoin EF, Maitra A, Rajapakse V, King C, Jacobetz MA, Ross S, Conrads TP, Veenstra TD, Hitt BA, et al. 2003. Preinvasive and invasive ductal pancreatic cancer and its early detection in the mouse. Cancer Cell 4: 437-450.

Hingorani SR, Wang L, Multani AS, Combs C, Deramaudt TB, Hruban RH, Rustgi AK, Chang S, Tuveson DA. 2005. Trp53 $3^{R 172 H}$ and Kras ${ }^{G 12 D}$ cooperate to promote chromosomal instability and widely metastatic pancreatic ductal adenocarcinoma in mice. Cancer Cell 7: $469-483$.
Hockemeyer D, Wang H, Kiani S, Lai CS, Gao Q, Cassady JP, Cost GJ, Zhang L, Santiago Y, Miller JC, et al. 2011. Genetic engineering of human pluripotent cells using TALE nucleases. Nat Biotechnol 29: 731-734.

Huang P, Xiao A, Zhou M, Zhu Z, Lin S, Zhang B. 2011. Heritable gene targeting in zebrafish using customized TALENs. Nat Biotechnol 29: 699-700.

Hung KE, Maricevich MA, Richard LG, Chen WY, Richardson MP, Kunin A, Bronson RT, Mahmood U, Kucherlapati R. 2010. Development of a mouse model for sporadic and metastatic colon tumors and its use in assessing drug treatment. Proc Natl Acad Sci 107: 1565-1570.

Ijichi H, Chytil A, Gorska AE, Aakre ME, Fujitani Y, Fujitani S, Wright CV, Moses HL. 2006. Aggressive pancreatic ductal adenocarcinoma in mice caused by pancreas-specific blockade of transforming growth factorbeta signaling in cooperation with active Kras expression. Genes Dev 20: $3147-3160$

Isalan M, Klug A, Choo Y. 2001. A rapid, generally applicable method to engineer zinc fingers illustrated by targeting the HIV-1 promoter. Nat Biotechnol 19: 656-660.

Jackson EL, Willis N, Mercer K, Bronson RT, Crowley D, Montoya R, Jacks T, Tuveson DA. 2001. Analysis of lung tumor initiation and progression using conditional expression of oncogenic K-ras. Genes Dev 15: 3243 3248 .

Ji H, Ramsey MR, Hayes DN, Fan C, McNamara K, Kozlowski P, Torrice C, Wu MC, Shimamura T, Perera SA, et al. 2007. LKB1 modulates lung cancer differentiation and metastasis. Nature 448: 807-810.

Johnson L, Mercer K, Greenbaum D, Bronson RT, Crowley D, Tuveson DA, Jacks T. 2001. Somatic activation of the K-ras oncogene causes early onset lung cancer in mice. Nature 410: 1111-1116.

Joung JK, Sander JD. 2012. TALENs: A widely applicable technology for targeted genome editing. Nat Rev Mol Cell Biol 14: 49-55.

Kim JH, Lee SR, Li LH, Park HJ, Park JH, Lee KY, Kim MK, Shin BA, Choi SY. 2011. High cleavage efficiency of a $2 \mathrm{~A}$ peptide derived from porcine teschovirus-1 in human cell lines, zebrafish and mice. PLoS One 6: e18556.

Kinross KM, Montgomery KG, Kleinschmidt M, Waring P, Ivetac I, Tikoo A, Saad M, Hare L, Roh V, Mantamadiotis T, et al. 2012. An activating Pik3ca mutation coupled with Pten loss is sufficient to initiate ovarian tumorigenesis in mice. J Clin Invest 122: 553-557.

Kirsch DG, Dinulescu DM, Miller JB, Grimm J, Santiago PM, Young NP, Nielsen GP, Quade BJ, Chaber CJ, Schultz CP, et al. 2007. A spatially and temporally restricted mouse model of soft tissue sarcoma. Nat Med 13: 992-997.

Kitayama H, Tsujimura T, Matsumura I, Oritani K, Ikeda H, Ishikawa J, Okabe M, Suzuki M, Yamamura K, Matsuzawa Y, et al. 1996. Neoplastic transformation of normal hematopoietic cells by constitutively activating mutations of c-kit receptor tyrosine kinase. Blood 88: 995-1004.

Lakso M, Sauer B, Mosinger B Jr, Lee EJ, Manning RW, Yu SH, Mulder KL, Westphal H. 1992. Targeted oncogene activation by site-specific recombination in transgenic mice. Proc Natl Acad Sci 89: 6232-6236.

Lang GA, Iwakuma T, Suh Y-A, Liu G, Rao VA, Parant JM, Valentin-Vega YA, Terzian T, Caldwell LC, Strong LC, et al. 2004. Gain of function of a p53 hot spot mutation in a mouse model of Li-Fraumeni syndrome. Cell 119: 861-872.

Lombardo A, Genovese P, Beausejour CM, Colleoni S, Lee YL, Kim KA, Ando D, Urnov FD, Galli C, Gregory PD, et al. 2007. Gene editing in human stem cells using zinc finger nucleases and integrase-defective lentiviral vector delivery. Nat Biotechnol 25: 1298-1306.

Lyons SK, Patrick PS, Brindle KM. 2013. Imaging mouse cancer models in vivo using reporter transgenes. Cold Spring Harb Protoc doi:10. 1101/pdb.top069864.

Maeder ML, Thibodeau-Beganny S, Osiak A, Wright DA, Anthony RM, Eichtinger M, Jiang T, Foley JE, Winfrey RJ, Townsend JA, et al. 2008. Rapid "open-source" engineering of customized zinc-finger nucleases for highly efficient gene modification. Mol Cell 31: 294-301.

Martin P, Albagli O, Poggi MC, Boulukos KE, Pognonec P. 2006. Development of a new bicistronic retroviral vector with strong IRES activity. BMC Biotechnol 6: 4.

Mercer K, Giblett S, Green S, Lloyd D, Darocha Dias S, Plumb M, Marais R, Pritchard C. 2005. Expression of endogenous oncogenic V600EB-raf 
induces proliferation and developmental defects in mice and transformation of primary fibroblasts. Cancer Res 65: 11493-11500.

Meuwissen R, Linn SC, Van Der Valk M, Mooi WJ, Berns A. 2001. Mouse model for lung tumorigenesis through Cre/lox controlled sporadic activation of the K-Ras oncogene. Oncogene 20: 6551-6558.

Meyer M, De Angelis MH, Wurst W, Kuhn R. 2010. Gene targeting by homologous recombination in mouse zygotes mediated by zinc-finger nucleases. Proc Natl Acad Sci 107: 15022-15026.

Moehle EA, Rock JM, Lee YL, Jouvenot Y, Dekelver RC, Gregory PD, Urnov FD, Holmes MC. 2007. Targeted gene addition into a specified location in the human genome using designed zinc finger nucleases. Proc Natl Acad Sci 104: 3055-3060.

Murphy DJ, Junttila MR, Pouyet L, Karnezis A, Shchors K, Bui DA, BrownSwigart L, Johnson L, Evan GI. 2008. Distinct thresholds govern Myc's biological output in vivo. Cancer Cell 14: 447-457.

Mussolino C, Cathomen T. 2012. TALE nucleases: Tailored genome engineering made easy. Curr Opin Biotechnol 23: 644-650.

Nakai N, Ishikawa T, Nishitani A, Liu N-N, Shincho M, Hao H, Isozaki K, Kanda T, Nishida T, Fujimoto J, et al. 2007. A mouse model of a human multiple GIST family with KIT-Asp820Tyr mutation generated by a knock-in strategy. J Pathol 214: 302-311.

Olive KP, Tuveson DA, Ruhe ZC, Yin B, Willis NA, Bronson RT, Crowley D, Jacks T. 2004. Mutant p53 gain of function in two mouse models of Li-Fraumeni syndrome. Cell 119: 847-860.

Pattanayak V, Ramirez CL, Joung JK, Liu DR. 2011. Revealing off-target cleavage specificities of zinc-finger nucleases by in vitro selection. Nat Methods 8: 765-770.

Perez-Mancera PA, Guerra C, Barbacid M, Tuveson DA. 2012. What we have learned about pancreatic cancer from mouse models. Gastroenterology 142: 1079-1092.

Pollock R, Giel M, Linher K, Clackson T. 2002. Regulation of endogenous gene expression with a small-molecule dimerizer. Nat Biotechnol 20: 729-733.

Rémy S, Tesson L, Ménoret S, Usal C, Scharenberg AM, Anegon I. 2009. Zinc-finger nucleases: A powerful tool for genetic engineering of animals. Transgenic Res 19: 363-371.

Ramirez CL, Certo MT, Mussolino C, Goodwin MJ, Cradick TJ, Mccaffrey AP, Cathomen T, Scharenberg AM, Joung JK. 2012. Engineered zinc finger nickases induce homology-directed repair with reduced mutagenic effects. Nucleic Acids Res 40: 5560-5568.

Reyon D, Khayter C, Regan MR, Joung JK, Sander JD. 2012a. Engineering designer transcription activator-like effector nucleases (TALENs) by REAL or REAL-Fast assembly. Curr Protoc Mol Biol Chapter 12: 12.15.1-12.15.14. doi: 10.1002/0471142727.mb1215s100.
Reyon D, Tsai SQ, Khayter C, Foden JA, Sander JD, Joung JK. 2012b. FLASH assembly of TALENs for high-throughput genome editing. Nat Biotechnol 30: 460-465.

Sander JD, Dahlborg EJ, Goodwin MJ, Cade L, Zhang F, Cifuentes D, Curtin SJ, Blackburn JS, Thibodeau-Beganny S, Qi Y, et al. 2011a. Selectionfree zinc-finger-nuclease engineering by context-dependent assembly (CoDA). Nat Methods 8: 67-69.

Sander JD, Maeder ML, Joung JK. 2011b. Engineering designer nucleases with customized cleavage specificities. Curr Protoc Mol Biol Chapter 12: Unit12 13.

Smith HW, Muller WJ. 2013. Transgenic mouse models-A seminal breakthrough in oncogene research. Cold Spring Harb Protoc doi:10.1101/ pdb.top069765.

Snyder EL, Watanabe H, Magendantz M, Hoersch S, Chen TA, Wang DG, Crowley D, Whittaker CA, Meyerson M, Kimura S, et al. 2013. Nkx2-1 represses a latent gastric differentiation program in lung adenocarcinoma. Mol Cell 50: 185-199.

Sommer G, Agosti V, Ehlers I, Rossi F, Corbacioglu S, Farkas J, Moore M, Manova K, Antonescu CR, Besmer P. 2003. Gastrointestinal stromal tumors in a mouse model by targeted mutation of the Kit receptor tyrosine kinase. Proc Natl Acad Sci 100: 6706-6711.

Thomas KR, Capecchi MR. 1987. Site-directed mutagenesis by gene targeting in mouse embryo-derived stem cells. Cell 51: 503-512.

Tikoo A, Roh V, Montgomery KG, Ivetac I, Waring P, Pelzer R, Hare L, Shackleton M, Humbert P, Phillips WA. 2012. Physiological levels of Pik3caH1047R mutation in the mouse mammary gland results in ductal hyperplasia and formation of ER $\alpha$-positive tumors. PLoS One 7: e36924.

Wang J, Abate-Shen C. 2014. Analyses of tumor suppressor genes in germline mouse models of cancer. Cold Spring Harb Protoc doi:10.1101/pdb. top069773.

Weber E, Gruetzner R, Werner S, Engler C, Marillonnet S. 2011. Assembly of designer TAL effectors by Golden Gate cloning. PLoS One 6: e19722.

Young NP, Crowley D, Jacks T. 2011. Uncoupling cancer mutations reveals critical timing of p53 loss in sarcomagenesis. Cancer Res 71: 4040-4047.

Yuan W, Stawiski E, Janakiraman V, Chan E, Durinck S, Edgar KA, Kljavin NM, Rivers CS, Gnad F, Roose-Girma M, et al. 2012. Conditional activation of $P i k 3 \mathrm{ca}^{\text {H1047R }}$ in a knock-in mouse model promotes mammary tumorigenesis and emergence of mutations. Oncogene 32: 318-326.

Zhu H, Acquaviva J, Ramachandran P, Boskovitz A, Woolfenden S, Pfannl R, Bronson RT, Chen JW, Weissleder R, Housman DE, et al. 2009. Oncogenic EGFR signaling cooperates with loss of tumor suppressor gene functions in gliomagenesis. Proc Natl Acad Sci 106: 2712-2716. 


\section{Genetically Engineered Knock-In and Conditional Knock-In Mouse Models of Cancer}

Amy Rappaport and Leisa Johnson

Cold Spring Harb Protoc; doi: 10.1101/pdb.top069799

\begin{tabular}{rc}
$\begin{array}{r}\text { Email Alerting } \\
\text { Service }\end{array}$ & Receive free email alerts when new articles cite this article - click here. \\
\hline $\begin{array}{c}\text { Subject } \\
\text { Categories }\end{array}$ & $\begin{array}{c}\text { Browse articles on similar topics from Cold Spring Harbor Protocols. } \\
\text { Analysis of Gene Expression (197 articles) } \\
\text { Analysis of Gene Expression, general (129 articles) } \\
\text { Genetics, general (374 articles) } \\
\text { Mouse (437 articles) } \\
\text { Use of Reporter Genes (124 articles) }\end{array}$ \\
\hline
\end{tabular}

